\title{
Using network pharmacology and molecular docking to explore the underlying anti-inflammatory mechanism of Wuyao-Danshen to treat endometriosis
}

\author{
Jie Zhu, Xiaoou Xue, Zhiping He, Jiawei Zhang, Haiyun Sun \\ Department of Gynaecology, Dongzhimen Hospital of Beijing University of Chinese Medicine, Beijing, China \\ Contributions: (I) Conception and design: J Zhu; (II) Administrative support: H Sun, Z He; (III) Provision of study materials or patients: X Xue; (IV) \\ Collection and assembly of data: J Zhu; (V) Data analysis and interpretation: J Zhang; (VI) Manuscript writing: All authors; (VII) Final approval of \\ manuscript: All authors. \\ Correspondence to: Haiyun Sun. Dongzhimen Hospital of Beijing University of Chinese Medicine, Dongcheng District, Beijing, China. \\ Email: sunhaiyun1979@163.com.
}

Background: This study sought to explore the anti-inflammatory mechanism of Wuyao (radix linderae)Danshen (salviae miltiorrhiza) in endometriosis (EMS) based on network pharmacology and molecular docking.

Methods: The active constituents of Wuyao-Danshen were collected and identified using the Traditional Chinese Medicine Systems Pharmacology Database, and used to predict and identify the protein targets. The EMS targets and anti-inflammatory targets were obtained from Genecards, Online Mendelian Inheritance in Man, and Drugbank. The Search Tool for the Retrieval of Interacting Genes/Proteins database was used to analyze the protein interactions (PPIs) and core targets, and a target PPI network was constructed by importing the software of Cytoscape. The Metascape database was used to conduct enrichment analyses of the Gene Ontology (GO) functions and the Kyoto Encyclopedia of Genes and Genomes (KEGG) signaling pathways for the key anti-inflammatory targets of EMS. Finally, Autodock Vina software was used to verify the results of the active ingredients and key anti-inflammatory targets.

Results: There were 8 active components in Wuyao, 65 in Danshen, and 591 corresponding protein targets in Danshen, and 375 in Wuyao, including luteolin, quercetin, vancomyl alcohol, and salvianol. One thousand and six hundred eighty-nine disease targets, 1,216 anti-inflammatory targets, and 144 key anti-inflammatory targets were identified, including the (signal transduction and transcriptional activator 3) STAT3, phosphatidyl inositol-3 kinase regulates subunit 1 (PIK3R1) and mitogen-activated protein kinase 1 (MAPK1) protein kinase B. Three hundred and fifty-three biological processes (BPs), 21 cellular components, and 25 molecular functions (MFs) were enriched with GO functions, and 108 KEGG pathways were enriched and analyzed, including the $M A P K$ and $P I 3 K$-Akt signaling pathways. Molecular docking confirmed that luteolin, coumarin, and quercetin could bind to the key target proteins (i.e., STAT3, PIK3R1, and MAPK1).

Conclusions: Based on network pharmacology and molecular docking, Wuyao-Danshen was found to act on EMS through anti-inflammatory targets and related signaling pathways. Our findings provide a basis for further research.

Keywords: Network pharmacology; molecular docking; Wuyao-Danshen; endometriosis (EMS); antiinflammatory

Submitted Dec 14, 2021. Accepted for publication Feb 22, 2022.

doi: $10.21037 / \mathrm{atm}-22-419$

View this article at: https://dx.doi.org/10.21037/atm-22-419 


\section{Introduction}

Endometriosis (EMS) is a common gynecological disorder characterized by the implantation of endometrial tissue outside the uterus, resulting in chronic inflammation, pain, and infertility (1). EMS is a refractory disease with extensive lesions, diverse forms, and it is extremely invasive and recurrent. It affects $10-15 \%$ of women of childbearing age in their lifetime (2). The incidence of EMS has increased in recent years, and seriously affects women's health and quality of life. EMS has a long history, but its exact pathogenesis is not yet known. The long-term abnormal inflammatory environment in the human body is an important factor in the development and progression of the disease, and the most typical histological features are local inflammation and peritoneal fibrosis (3). Western medicine treatment methods focus on estrogen suppression, including gonadal hormone analogues and progesterone (4), or surgical treatment if necessary. However, estrogen suppression has many side effects, and the condition repeats after drug withdrawal, and removing the lesion by surgery does not provide a cure, which is often unacceptable to patients. The traditional Chinese medicine (TCM) treatment has no inhibitory effect on the pituitary gland and ovary, does not interfere with the normal menstrual cycle, has an obvious curative effect (i.e., relieves pain, controls the development of lesions, and regulates menstruation), and does not affect pregnancy.

The main treatment for EMS in TCM is to activate blood and remove blood stasis. Professor Wang Ziyu, a nationally renowned veteran TCM practitioner, also believes that blood stasis is the main pathology of EMS, and the Qi stagnation and cold clotting of blood stasis is common. Wu Dan pill is a famous formula of Professor Wang to treat EMS by invigorating blood, benefiting kidney, dispersing cold and relieving pain. It consists of more than 10 herbal flavors, including Wu Yao, Dan Shen, Tao Ren, Red Peony, Curcuma longa, Leech, Yuan Hu, Cinnamon, and Chuan Jie. Wuyao-Danshen is combination as the monarch medicine, which plays a key role.

Network pharmacology (5) is a new type of biological system network analysis that is based on systems biology theory. It seeks to understand the nature of diseases from the holistic perspective of network balance, and systematically explores the efficacy and mechanisms of TCM with multicomponent, multi-target, and multi-mechanism effects. A network pharmacology research method was used in this study to explore the effect of the inflammatory reaction of the wudan pill gentleman medicine (i.e., radix linderae- salvia miltiorrhiza), and the potential anti-inflammatory mechanism of EMS. Molecular docking technology, key efficacy components, and docking target validation were used to further elucidate the clinical curative effect of the medicine and the underlying mechanism.

\section{Methods}

\section{Materials}

Table 1 sets out the databases, analysis software, and analysis platform information used in this experiment.

\section{Study methods}

\section{Screening of drug active ingredients and corresponding} targets

The Traditional Chinese Medicine Systems Pharmacology Database (TCMSP) database was searched using the following terms: "Radix linderae" and "salvia miltiorrhiza", with an OB (oral availability) $\geq 30 \%$, and DL (similarity of patent medicine) $\geq 0.18$ to determine the active compound composition information for "Radix linderae-Salvia miltiorrhiza". Additionally, PubChem was searched for details of the compound composition of the TCM not included in the TCMSP database. Next, swisStar prediction was used to predict potential target proteins of the drug compounds. Then, after logging into the Uniprot database, the biological species was limited to "human", and the data type was set to be reviewed according to the full name of the candidate target, and the corresponding gene name and Uniprot KB (UniProt Knowledgebase) of the target were obtained.

\section{Collection of EMS and anti-inflammatory targets}

The GeneCards database was searched using the following keywords: "Endometriosis" and "anti-inflammatory". Correlation scores $>20$ were set to retrieve information on the related genes. The Online Mendelian Inheritance in Man (OMIM), DRUGBANK, Therapeutic Target Database (TTD), and DISGENET databases were then searched using the same keywords. The targets of the 5 databases were uploaded to the UniProt database to identify the corresponding gene information and correct the targets. Next, the intersection of the EMS target with the anti-inflammatory related targets and compound targets was obtained to identify the common anti-inflammatory targets in the treatment of EMS with Wuyao-Danshen. The study was conducted in accordance with the Declaration of 
Table 1 Database and software information used in the experiment

\begin{tabular}{ll}
\hline Name & Website \\
\hline TCMSP & https://tcmspw.com/tcmsp.php \\
PubChem & https://www.ncbi.nlm.nih.gov/pccompound \\
Swiss Target & http://www.swisstargetprediction.ch/ \\
Prediction & \\
Uniprot & https://www.un iprot.org/ \\
GeneCards & https://www.genecards.org/ \\
OMIM & https://omim.org/ \\
DRUGBANK & https://go.drugbank.com/ \\
TTD & http://db.idrblab.net/ttd/ \\
DISGENET & https://www.genecards.org/ \\
STRING & www.string-db.org \\
Cytoscape & - \\
Venny & http://www.bioinformatics.com.cn \\
Metascape & https://metascape.org \\
Autodock vina & - \\
Autodock tool & - \\
Discovery & - \\
Python & - \\
Pymol & - \\
\hline
\end{tabular}

Helsinki (as revised in 2013).

\section{Analysis of common anti-inflammatory targets}

The obtained common anti-inflammatory targets were imported into the Search Tool for the Retrieval of Interacting Genes/Proteins (STRING) database in the form of Genes YMBOL to analyze the interaction between proteins and obtain the key anti-inflammatory targets for the treatment of EMS with Wuyao-Danshen. Dig name url TCMSP dug core targets and protein interaction (PPI) network diagram. The species was selected as "human", and the minimum interaction threshold was set as "high confidence $(\geq 0.9)$ ". To improve the confidence of the PPI, a combined score $>0.7$ was selected, and nodes (Node 1 , and Node 2), and the combined score information data were saved. Next, Cytoscape version 3.7.1 software was imported and a topology analysis was conducted using the NetworkAnalyzer plug-in. The core targets were screened according to the median centrality, compact density, and node connection degree.

\section{Gene Ontology (GO) enrichment and Kyoto Encyclopedia of Genes and Genomes (KEGG) pathway analysis}

Metascape (6) contains details of biological pathways and protein complexes, applies a powerful analytical pipeline to produce easily interpretable results, provides an annotated gene list, and rich and interactive resources, and supports the integrated analysis of multiple omics data sets. GO enrichment analysis and a KEGG pathway enrichment analysis were conducted to examine the potential role of "radix linderae-salvia miltiorrhiza" in the treatment of EMS target genes, and determine the corresponding molecular functions (MFs), biological processes (BPs), cellular compositions (CCs) and KEGG pathway-related data. To improve the confidence of the related pathways, the top 10 genes from a large proportion of genes enriched in the pathways were then screened, and data visualization was performed using the online platform of WeChat biogenesis to determine their main biological functions (BFs) and related signaling pathways.

\section{Molecular docking}

The core target protein structure was downloaded from the Protein Data Bank (PDB) database, and the water molecule was removed using PyMol1.8 software, and the original ligand was separated. After saving, it was imported into AutodockTools1.5.6 software for hydrogenation, total charge calculation, and atomic type setting, and saved in "PDBQT" format. The MOL2 structure of the core component (ligand) screened above was downloaded from the TCMSP database, and the rotable key was set by Autodock Tools and saved as a file in "PDBQT" format. Finally, molecular docking was performed by AutodockVina1.1.2 software. PyMol software visualizes the docking results and creates docking interaction model diagrams.

\section{Results}

\section{Collection of drug active ingredients and corresponding targets}

Through TCMSP and PubChem, "radix linderae-salvia miltiorrhiza" compounds were retrieved, and 8 radix linderae and 65 salvia miltiorrhiza effective compounds were identified. The Wiss Target Prediction database predicted and identified 591 salvia miltiorrhiza and 375 aconite protein 
Table 2 Main components of radix linderae-salvia miltiorrhiza

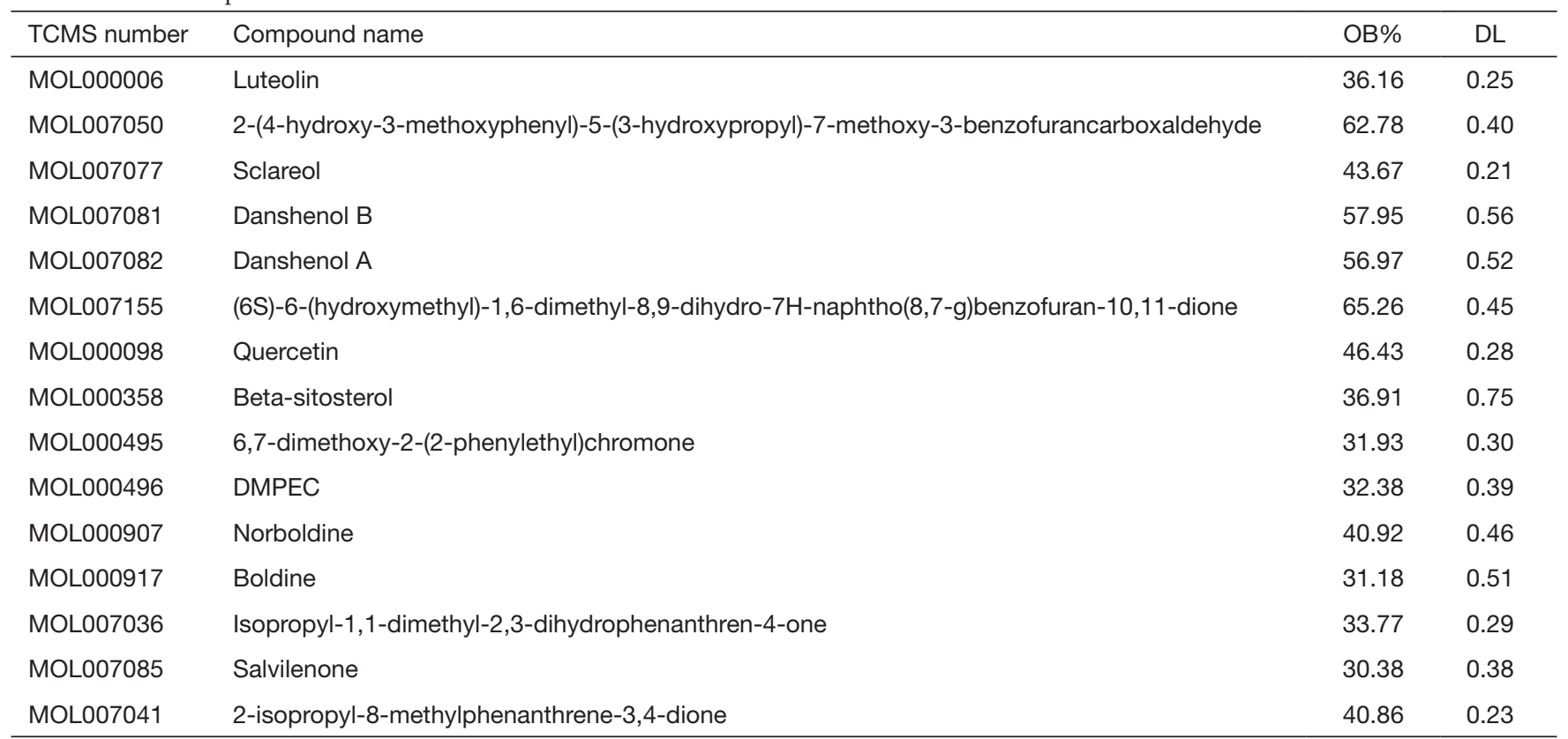

$\mathrm{OB}$, oral availability; DL, similarity of patent medicine.

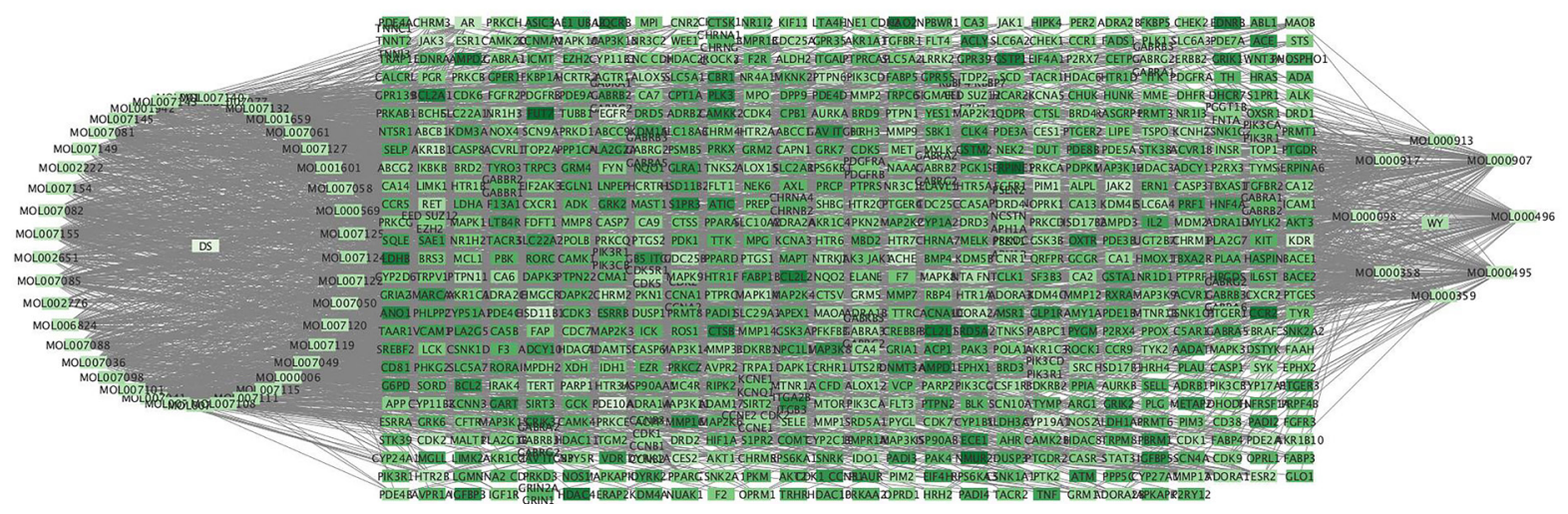

Figure 1 Drug-compound-target network diagram.

targets corresponding to the active ingredients (see Table 2). The drug active components and their corresponding targets were imported into Cytoscape 3.7.1 to construct a drugcomponent-target network diagram. The network comprised 271 nodes, including 122 active ingredient nodes, 81 target nodes, and 1,244 edges. As Figure 1 shows, "radix linderaesalvia miltiorrhiza" mainly acts through multiple components corresponding to multiple targets.

\section{Collection of disease-related targets}

DISGENET, DRUGBANK, GeneCards, OMIM, and
TTD were used to search and obtain EMS target genes. Next, the 1,689 target genes were converted into UNIPROt by UNIPROt. A total of 1,216 anti-inflammatory targets were identified using the same method. A total of 559 targets were identified from the intersection of EMS with anti-inflammatory targets, and 144 targets were identified from the intersection of EMS with effective compound targets (see Figure 2).

\section{PPI analysis of key targets}

Homo sapiens were selected to construct the PPI network 


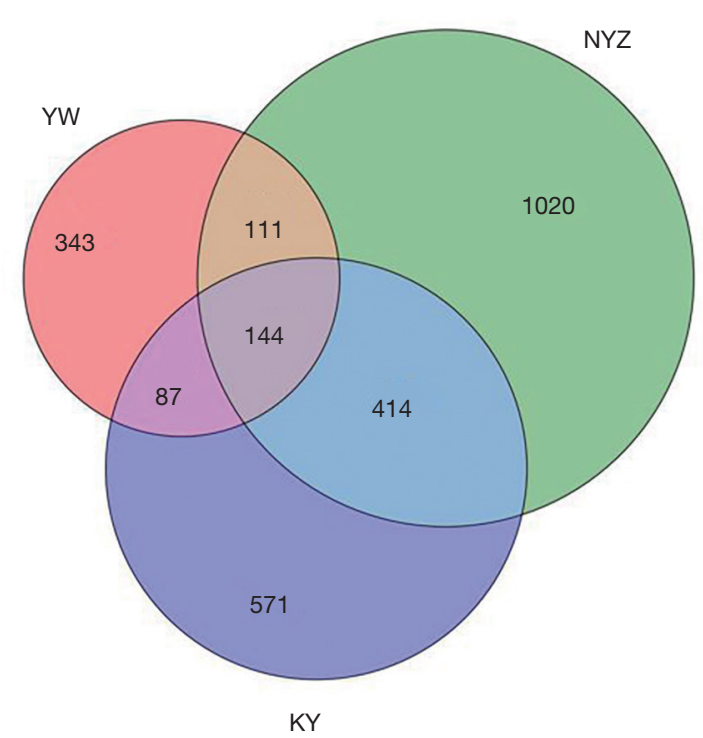

Figure 2 Venn diagram of the intersection of drug-EMS - antiinflammatory. EMS, endometriosis; NYZ, Endometriosis; YW, drug (wuyao-danshen); KY, anti-inflammatory.

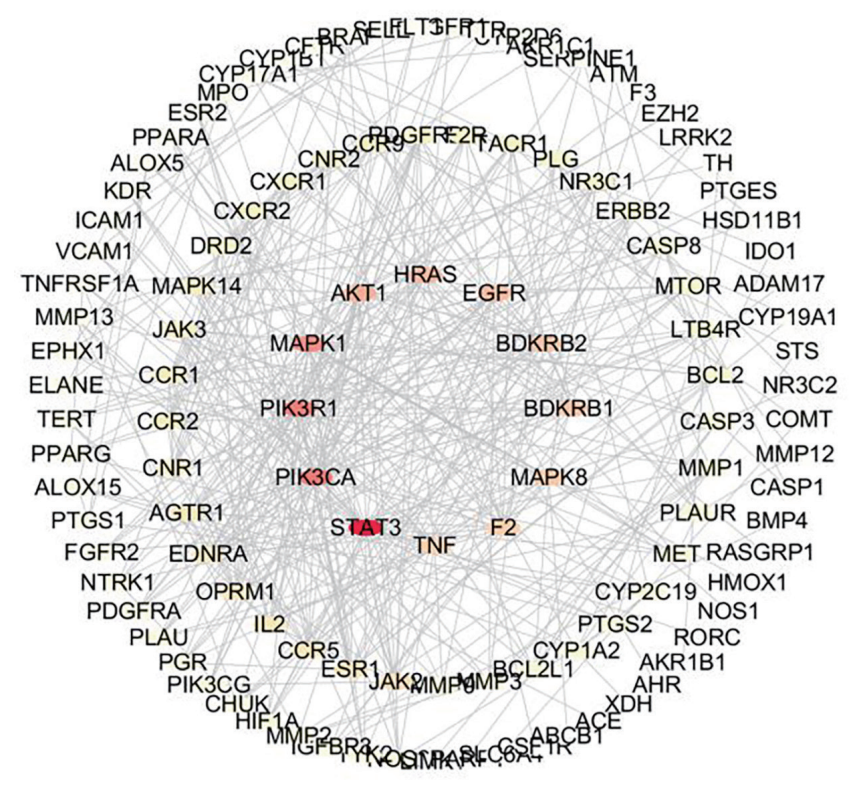

Figure 3 Interaction network diagram of key anti-inflammatory target proteins.

map, which was visualized using Cytoscape software. The nodes in the network map represented the protein molecules of the anti-inflammatory targets, and the edges represented the relationship between each target. The color in the figure represents the value of the degree of the anti- inflammatory target; the intensity of the color (from light to dark) indicates the degree value of the anti-inflammatory target (from smaller to larger). The higher the de-GRE values of signal transduction and transcriptional activator 3 (STAT3), alpha catalytic subunit of phosphoinositol-3-kinase (PIK3CA), PIK3R1, MAPK1, AKT1, (GTPase Hras) HRAS, epidermal growth factor receptor $(E G F R)$ and Bradykinin receptor $\mathrm{B} 1$ ( $B D K R B 1)$, the more likely they are to be the key anti-inflammatory targets of endopathy (see Figure 3).

\section{GO and KEGG patbway enrichment analyses}

A GO enrichment analysis was conducted to describe the gene functions, and a KEGG pathway enrichment analysis was conducted to identify the significantly enriched signal pathways and explore the BPs and signal pathways of EMS treatment by radix linderae-salvia miltiorrhiza. The Metascape platform was used for the GO and KEGG pathway analyses $(\mathrm{P}<0.05)$, and a pathway annotation analysis was combined with the database. There were 399 GO items $(\mathrm{P}<0.05)$, including $353 \mathrm{BP}$ items, $21 \mathrm{CC}$ items, and $25 \mathrm{MF}$ items. The top 10 items of each component were imported into the bioinformation platform for visualization (see Figure 4). The BP entries occurred before the response to lipopolysaccharide (the reaction), the response to the molecule of bacterial origin (the bacteria source molecular reaction), and the positive regulation of the response to external stimulus (external stimulating reaction positive adjustment). At present, the $2 \mathrm{CC}$ strips were the Caveola and Plasman embrace raft. MF article 2 was currently the transmembrane receptor protein tyrosine kinase activity transmembrane receptor protein kinase activity (transmembrane receptor protein kinase activity). The color intensity represents the size of $\mathrm{P}$; the darker the color, the smaller the $\mathrm{P}$. The bubble size represents the number of enrichment targets in this pathway; the larger the number of points, the greater the number. There were 108 KEGG signaling pathways. The mitogen-activated protein kinase $(M A P K)$ signaling Pathway and Phosphoinositide 3-kinase-Protein kinase $\mathrm{B}(P I 3 K-A K T)$ signaling pathway were the most significant (see Figure 5).

\section{Molecular docking}

To further validate the effect of the wudan pill gentleman medicine (i.e., radix linderae-salvia miltiorrhiza) in the treatment of EMS candidate compounds, the compounds 78 and participation key pathways targets before docking, 

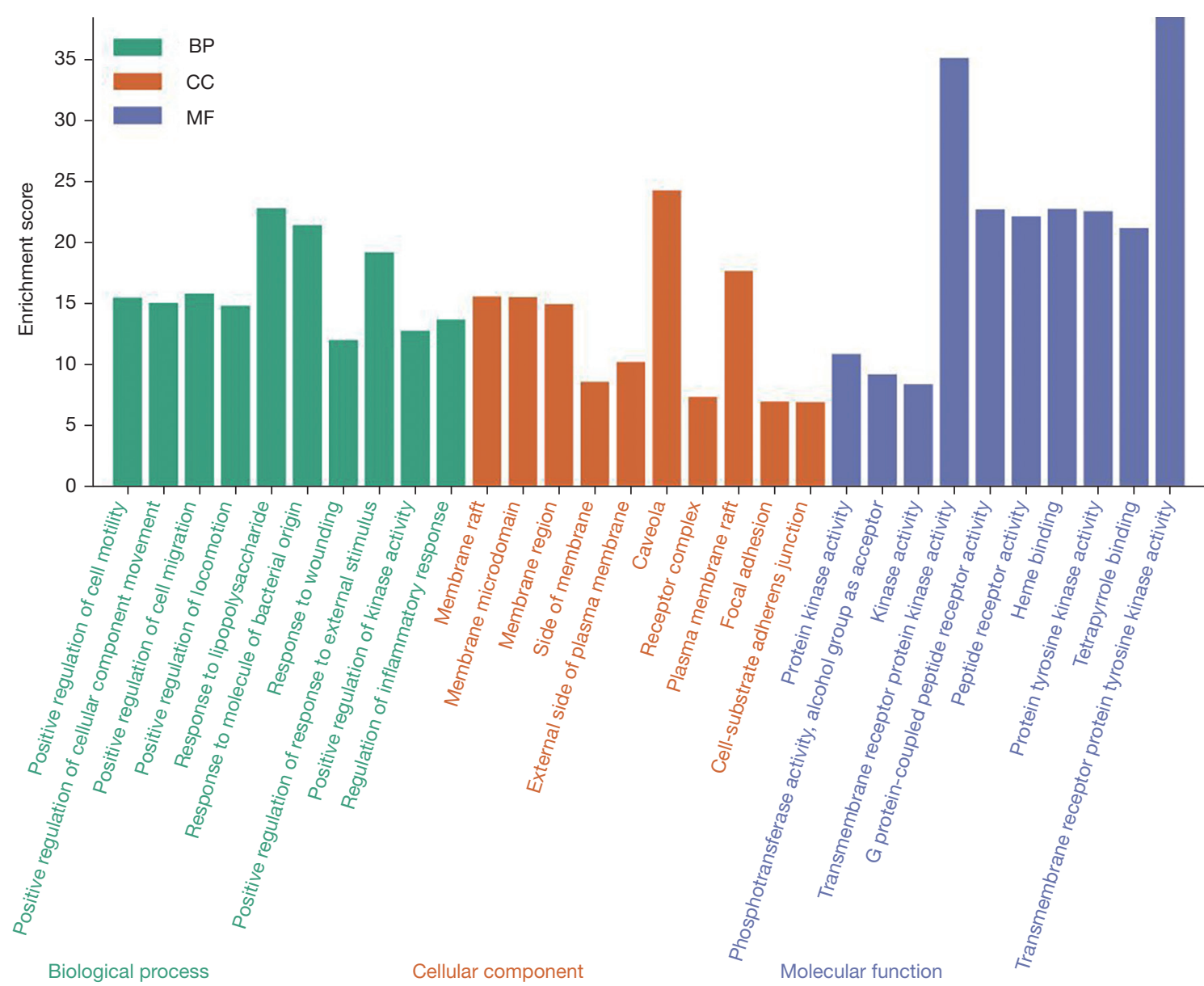

Figure 4 Histogram of GO enrichment at key targets. GO, Gene Ontology; BP, biological process; CC, cellular component; MF, molecular function.

found the lowest binding energy, $-5.0 \mathrm{~kJ} \mathrm{~mol}, 1$ or less, results demonstrate the validity of efficacy of molecular docking with the protein, the lower the binding energy, show that molecular and protein binding ability is poor (see Table 3). The docking results of luteolin, sclareol, quercetin, and the potential target proteins of STAT3 (PDB:6NUQ), PIK3R1 (PDB:6PYR), and MAPK1 (PDB:6G8X) were visualized using PyMol software (see Figure 6). The docking analysis successfully predicted that luteolin, vanillyl alcohol, and quercetin bind well to the activity sites of the 3 target proteins. These results further prove that these 3 proteins can be used as therapeutic targets of luteolin, vanillyl alcohol, and quercetin in the inflammatory response of EMS.

\section{Discussion}

EMS is an immune inflammatory disease, which is mainly treated by surgery and drug therapy, but it produces adverse reactions, and EMS is prone to recurrence. Research on long-term chronic disease management needs to be conducted. TCM has played a positive role in safe guarding the fertility of EMs patients by taking the advantages of holistic concept and evidence-based treatment in the treatment of EMs, but the exact mechanism of action is unknown and needs to be explored by applying modern research methods in order to take advantage of natural herbal medicines. Scholars at home and abroad have turned their attention to the development and use of TCM to identify safe and effective drugs. Ziyu's empirical prescription of wudan pills has been clinically proven to improve the symptoms of EMS patients. Many studies have shown that aconite $(7,8)$ has anti-inflammatory and analgesic effects, and salvia miltiorrhiza $(9,10)$ has antiinflammatory and blood-activating effects. which participate 


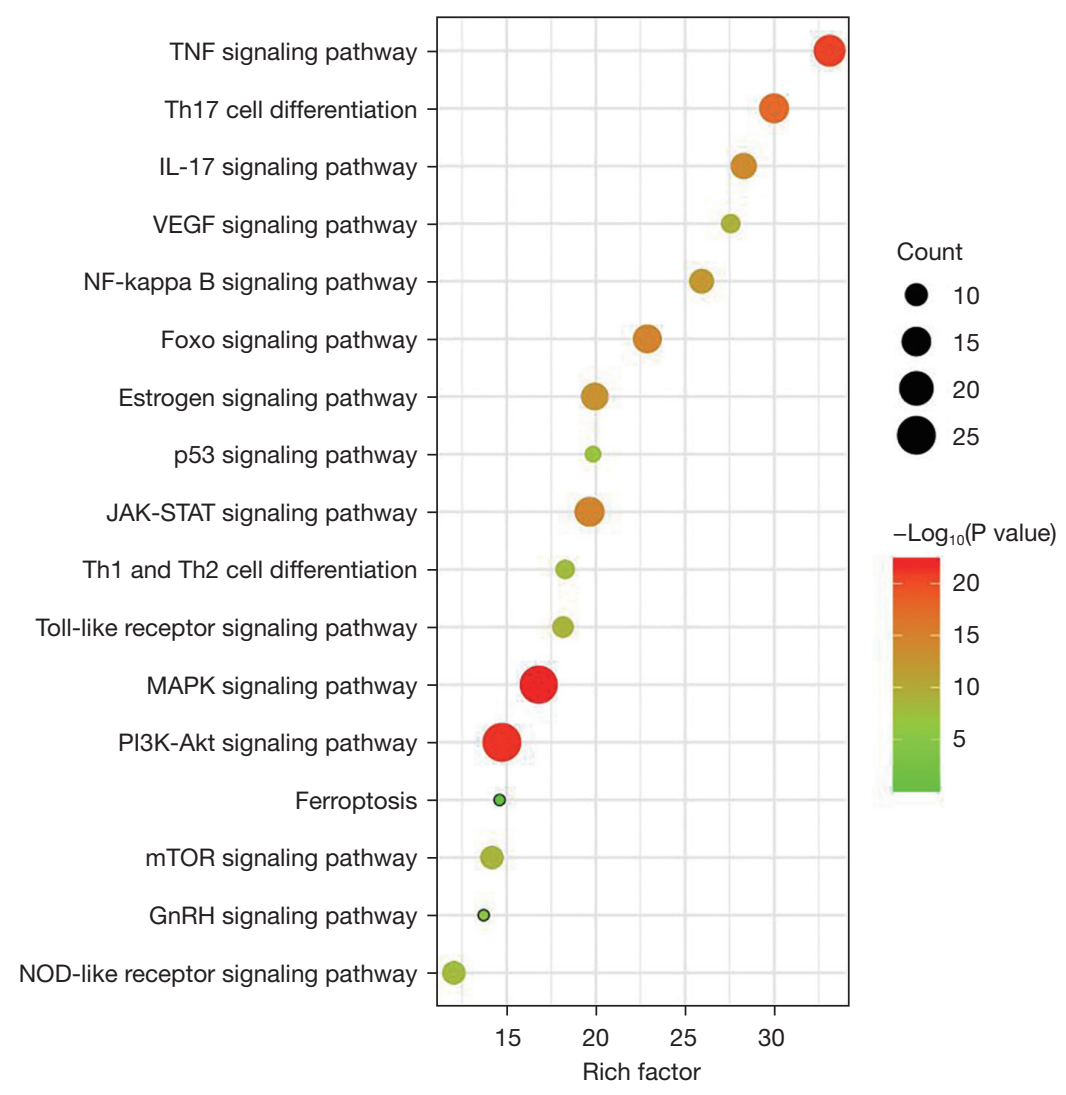

Figure 5 KEGG enrichment pathway analysis of key targets. KEGG, Kyoto Encyclopedia of Genes and Genomes.

Table 3 Binding capacity of core compounds to core proteins $\left(\mathrm{Kcal}^{\mathrm{m}} \mathrm{mol}^{-1}\right)$

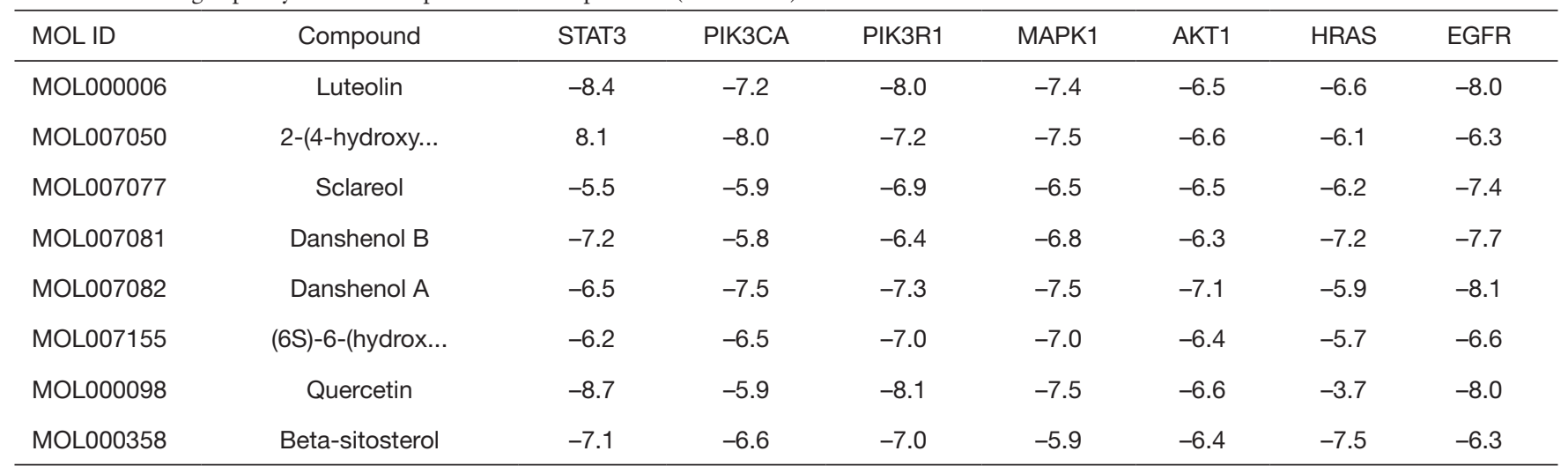

2-(4-hydroxy..., 2-(4-hydroxy-3-methoxyphenyl)-5-(3-hydroxypropyl)-7-methoxy-3-benzofurancarboxaldehyde; (6S)-6-(hydrox..., (6S)-6(hydroxymethyl)-1,6-dimethyl-8,9-dihydro-7H-naphtho(8,7-g)benzofuran-10,11-dione.

in the inflammatory response of EMS, and inhibit the growth of ectopic endometrium. Thus, this study explored and analyzed the anti-inflammatory mechanism of radix linderae-salvia miltiorrhiza in the treatment of EMS from an anti-inflammatory perspective.
In this study, effective active compounds, such as luteolin of salvia miltiorrhiza, vanillyl alcohol, salviol, and quercetin of aconitum, were identified in the TCMSP database. Luteolin (11) has anti-inflammatory and antioxidant effects, this study (12) has shown that luteolin affects the 


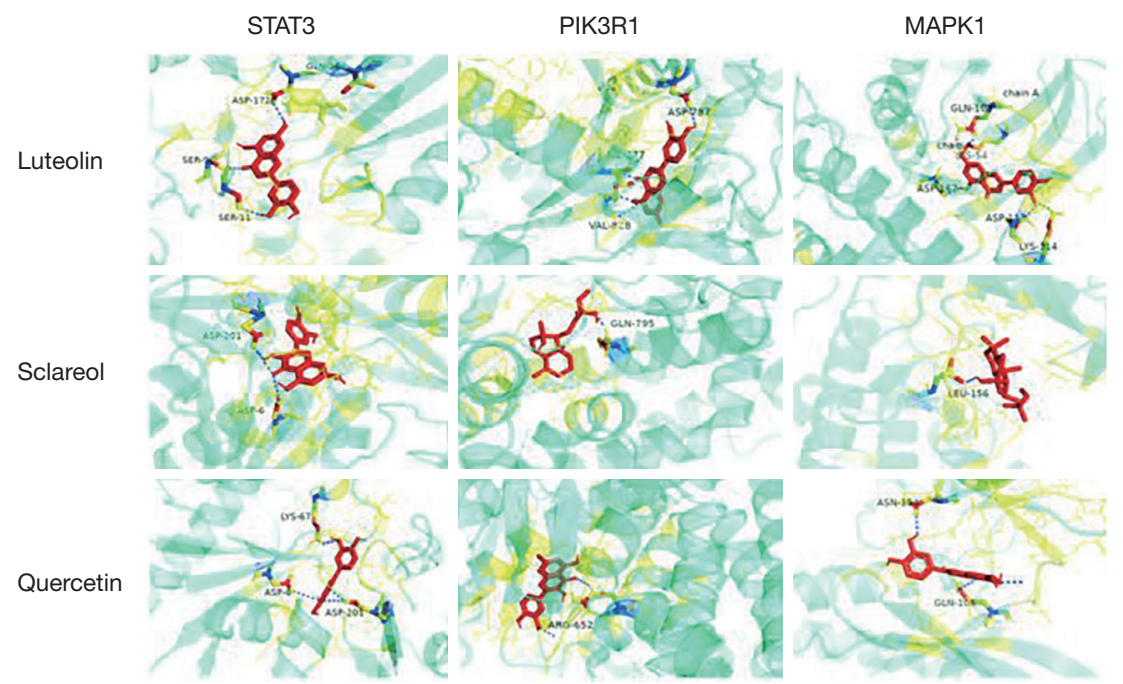

Figure 6 Molecular docking diagram of some key targets and active ingredients.

expression of inflammatory mediators. Quercetin is a natural flavonoid, and the study (13) has shown that it has antibacterial, anticancer, antioxidant, and anti-inflammatory effects. Tian et al. (14) found that quercetin inhibits End1/ E6E7 proliferation and promotes the apoptosis of EMS cells, and this inhibition is effective in the treatment of EMS. Some experiments have also shown that quercetin inhibits inflammation. Notably, Tang et al. (15) showed that quercetin inhibited the expression of NOD-like receptors family pyrin domain containing 3 (NLRP3) inflammasomerelated proteins in (Henrietta Lacks) HeLa cells in a dosedependent manner, thus inhibiting inflammation.

In this study, drug, disease and anti-inflammatory related targets were identified through the database number, and 144 anti-inflammatory targets for EMS treatment by radix linderae-salvia miltiorrhiza were identified by mapping their intersections with each other. The PPI results for the anti-inflammatory targets indicated that STAT3, PIK3CA, PIK3R1, MAPK1, AKT1, HRAS, EGFR, and BDKRB1 may be the key anti-inflammatory targets of black herb and salviae miltiorrhiza in EMS treatment.

STAT3 (16), a member of the STAT protein family, plays an important role in various physiological processes, such as cell growth, differentiation, immune function, and hematopoiesis, and its activation may promote the infiltration and metastasis of active endometrium by regulating a variety of cytokines and proteins. Research (17) has shown that STAT3 is over-activated in the ectopic endometrium of EMS patients, which may promote cell growth and resist apoptosis, angiogenesis, and invasion by regulating the expression of matrix metalloproteinase-2 $(M M P-2)$, matrix metalloproteinase-9 (MMP-9), and vascular endothelial growth factor $(V E G F)$. Another study (18) has found correlations between EMS combined with infertility and signal transduction and STAT3 gene polymorphism. Most studies of EMS (19) have noted that due to the continuous stimulation of external cytokines, activated STAT3 regulates the transcription of relevant downstream target genes, promoting the abnormal proliferation, invasion, and metastasis of ectopic endometrial cells, and participating in the formation of an inflammatory microenvironment, epithelial-mesenchymal transformation, extracellular matrix degradation, and other processes. When STAT3 in the cytoplasm is activated by Janus kinase $2(\mathcal{F} A K 2)$, it can enter the nucleus via the direct and indirect regulation of related genes and promote angiogenesis and induce cell proliferation, differentiation, or apoptosis. Some scholars (20) have found that the inhibition of the $7 A K 2 / S T A T 3$ signaling pathway has a positive effect on EMS treatment.

PIK3CA and PIK3R1 are common oncogenes, and it has been reported (21) that PIK3R1 has high frequency mutations in endometrial cancer, and the PI3K family plays a key role in the transduction of intracellular signals and the pathogenesis of inflammation, obesity, tumors, and immune diseases (22). The P110 catalytic subunit encoded by the PIK3CA gene and the P85 regulatory subunit encoded by the PIK3R1 gene mainly control important cellular activities, such as protein synthesis, cell growth and proliferation, angiogenesis, cell cycle, and cell survival. 
When PI3 kinase is phosphorylated, its level is regulated by phosphatase and tensin homolog (PTEN) phosphatase activity. The signal transmission activates $A K T$, which regulates the downstream effector activation of mechanistic target of rapamycin (mTOR). MTOR is a serine-threonine kinase that plays an important role in cell growth, proliferation, and regulation. In recent years, the study of EMS and endometrial cancer through the PI3K/Akt/mTOR pathway $(23,24)$ has become a popular area of research. The PI3K/Akt/mTOR pathway plays an important role in inflammatory diseases (25). Currently, very few studies have been conducted on the inflammatory response of EMS.

MAPK1 (26) is mainly involved in the regulation of cell proliferation, differentiation, growth, and apoptosis. The activation of the MAPK signaling pathway affects the activity of a variety of transcription factors, and thus regulates the expression of tumor necrosis factors $(T N F)$, interleukin-1 $(I L-1)$, interleukin-6 $(I L-6)$, and other inflammatory cells. Wang et al. (27) confirmed that the Shaofu Zhuyu Decoction downregulates the expression of tumor necrosis factor alpha $(T N F-\alpha), I L-6, I L-8$, nuclear factor kappa-light-chain-enhancer of activated B cells $(N F-\kappa B), M A P K$, Extracellular Signal Regulated Kinase (ERK), and $V E G F$, etc., through the $M A P K / E R K$ signaling pathway, which is mediated by estrogen, inhibits inflammatory factors and angiogenesis in ectopic tissues, and slows the occurrence and development of EMS. In the enrichment of KEGG pathways, MAPK1 is directly or indirectly involved in the TNF signaling pathways, kuohaonucleotide-binding oligomerization domain protein (NOD) receptors signaling pathways, and $M A P K$ signaling pathways, and forms a complex interaction relations of "wu medicine danshen active ingredient-anti-inflammatory target-signal path-endometriosis" network.

\section{Conclusions}

In this study, through the method of network pharmacology, it was found that the main active ingredients of radix linderae-salvia miltiorrhiza in the treatment of EMS inflammation may include luteolin, vanillyl alcohol, quercetin, and salviol $\mathrm{B}$. These active ingredients inhibit the inflammatory response of EMS by acting on STAT3, $P I K 3 R 1$, and MAPK1, and regulating the MAPK, PI3KAkt signaling pathway, $T N F, N F-K B$, and other signaling pathways. Additionally, molecular docking was also used to verify that the active drug ingredients have a strong binding force with the key targets. We further confirmed the anti-inflammatory mechanism of a multi-target, multipath, and coordinated treatment of radix linderae-salvia miltiorrhiza in the treatment of EMS, and provided a basis for subsequent experimental verification.

\section{Acknowledgments}

Funding: None.

\section{Footnote}

Conflicts of Interest: All authors have completed the ICMJE uniform disclosure form (available at https://atm. amegroups.com/article/view/10.21037/atm-22-419/coif). The authors have no conflicts of interest to declare.

Ethical Statement: The authors are accountable for all aspects of the work in ensuring that questions related to the accuracy or integrity of any part of the work are appropriately investigated and resolved. The study was conducted in accordance with the Declaration of Helsinki (as revised in 2013).

Open Access Statement: This is an Open Access article distributed in accordance with the Creative Commons Attribution-NonCommercial-NoDerivs 4.0 International License (CC BY-NC-ND 4.0), which permits the noncommercial replication and distribution of the article with the strict proviso that no changes or edits are made and the original work is properly cited (including links to both the formal publication through the relevant DOI and the license). See: https://creativecommons.org/licenses/by-nc-nd/4.0/.

\section{References}

1. Patel BG, Lenk EE, Lebovic DI, et al. Pathogenesis of endometriosis: Interaction between Endocrine and inflammatory pathways. Best Pract Res Clin Obstet Gynaecol 2018;50:50-60.

2. Lang J. The history, present situation and development of endometriosis. Chinese Journal of Practical Gynecology and Obstetrics 2020;36:193-6.

3. Wang XD, Zheng P. Research Progress on Immune Mechanism of Endometriosis. Int J Obstet Gynecol 2019;46:536-40.

4. Lindsay SF, Luciano DE, Luciano AA. Emerging therapy for endometriosis. Expert Opin Emerg Drugs 2015;20:449-61. 
5. Boezio B, Audouze K, Ducrot P, et al. Network-based Approaches in Pharmacology. Mol Inform 2017;36. doi: 10.1002/minf.201700048.

6. Zhou Y, Zhou B, Pache L, et al. Metascape provides a biologist-oriented resource for the analysis of systemslevel datasets. Nat Commun 2019;10:1523.

7. Zhang J, Luo R, Yang Y, et al. Study on anti-inflammatory and analgesic pharmacology of total alkaloid of Aconite. Chinese Journal of Hospital Pharmacy 2016;36:2187-90.

8. Wang Q, Hu M, Wang G. Study on analgesic and antiinflammatory effects of Aconite alkaloids. Chinese Journal of Traditional Chinese Medicine 2015;33:910-2.

9. Li Y, Liu J, Liang Y, et al. Anti-inflammatory effects of water-soluble components of Salvia miltiorrhiza. Heilongjiang Animal Husbandry and Veterinarian 2020:132-134+137.

10. Chai H, Qu H, Du J, et al. Progress in the combined application of Traditional Chinese medicine for promoting blood circulation and removing stasis and antiplatelet drugs(J/OL). Chinese Journal of Integrated Traditional and Western Medicine:1-4(2020-11-23). doi: 10.7661/ j.cjim.20200306.218.

11. Yang SC, Chen PJ, Chang SH, et al. Luteolin attenuates neutrophilic oxidative stress and inflammatory arthritis by inhibiting Raf1 activity. Biochem Pharmacol 2018;154:384-96.

12. Shi J, Wang S, Cao M, et al. Effects of luteolin on BMDM polarity and expression of inflammatory factors (J/OL). Chinese Journal of Nosocomiology 2020;(23):3568-73.

13. Zhu H. Therapeutic effect of quercetin on cervicitis in rats. Journal of Xinxiang Medical College 2019;36:417-20.

14. Tian J, Yao L, Su M, et al. Effects of quercetin combined with CADM1 on proliferation and apoptosis of endometriosis cells. Exploration on Rational Drug Use in China 2020;17:95-9.

15. Tang Y, Wang W, Chen R, et al. Role of NLRP3 inflammasome in quercetin induced apoptosis of cervical cancer HeLa cells. Modern Oncol 2021;29:5-9.

16. Okamoto M, Nasu K, Abe W, et al. Enhanced miR-210

Cite this article as: Zhu J, Xue X, He Z, Zhang J, Sun H. Using network pharmacology and molecular docking to explore the underlying anti-inflammatory mechanism of Wuyao-Danshen to treat endometriosis. Ann Transl Med 2022;10(4):198. doi: 10.21037/atm-22-419 expression promotes the pathogenesis of endometriosis through activation of signal transducer and activator of transcription 3. Hum Reprod 2015;30:632-41.

17. Cui Y, Li P, Hao S, et al. STAT3, MMPs and VEGF expression levels in patients with endometriosis. Maternal and Child Health Care in China 2019;34:250-2.

18. Juan Hu, Feng Ye. Correlation between endometriosis complicated with infertility and STAT3 gene polymorphism. Journal of Hainan Medical College 2016;22:948-50.

19. Zhu X, Han Y, Han Y, et al. Advances in the study of endometriosis related signaling pathways. Obstet Gynecol Int J 2019;46:370-3.

20. Chen J, Yang R, Liu H, et al. The role of JAK2/STAT3 signaling pathway in the pathogenesis of endometriosis. Tianjin Medical 2019;47:136-9.

21. Urick ME, Rudd ML, Godwin AK, et al. PIK3R1 (p85a) is somatically mutated at high frequency in primary endometrial cancer. Cancer Res 2011;71:4061-7.

22. Tan A, Luo R, Ruan P. miR-495 promotes apoptosis and inhibits proliferation in endometrial cells via targeting PIK3R1. Pathol Res Pract 2019;215:594-9.

23. Zhang $\mathrm{Y}, \mathrm{Li} \mathrm{W}, \mathrm{Li}$ J, et al. Effect of Juantong Decoction on egg white of PI3K/Akt/mTOR signaling pathway in endometriosis rats. Chinese Journal of Experimental Formulae 2019;25:24-30.

24. Barra F, Ferro Desideri L, Ferrero S. Inhibition of PI3K/ AKT/mTOR pathway for the treatment of endometriosis. Br J Pharmacol 2018;175:3626-7.

25. Ji Y, Gong G. Research progress on molecular mechanism of PI3K/Akt/mTOR pathway in inflammatory diseases. Pharm Res 2018;37:226-9.

26. Mormile R, Vittori G. MAPK signaling pathway and endometriosis: what is the link? Arch Gynecol Obstet 2013;287:837-8.

27. Wang J, Cui Y, Cheng Y, et al. Effect of Shaofu Zhuyu Decoction on MAPK/ERK signaling pathway in endometriosis rats. Chinese Journal of Pathophysiology 2019;35:181-7. 